\title{
Stability and Hopf Bifurcation Analysis for a Stage-Structured Predator-Prey Model with Discrete and Distributed Delays
}

\author{
Ruiqing Shi, ${ }^{1,2}$ Junmei Qi, ${ }^{2}$ and Sanyi Tang ${ }^{1}$ \\ ${ }^{1}$ College of Mathematics and Information Science, Shaanxi Normal University, Xian, Shaanxi 710062, China \\ ${ }^{2}$ School of Mathematics and Computer Science, Shanxi Normal University, Linfen, Shanxi 041004, China \\ Correspondence should be addressed to Ruiqing Shi; shirq1979@163.com
}

Received 26 April 2013; Accepted 17 September 2013

Academic Editor: Maoan Han

Copyright (C) 2013 Ruiqing Shi et al. This is an open access article distributed under the Creative Commons Attribution License, which permits unrestricted use, distribution, and reproduction in any medium, provided the original work is properly cited.

We propose a three-dimensional stage-structured predatory-prey model with discrete and distributed delays. By use of a new variable, the original three-dimensional system transforms into an equivalent four-dimensional system. Firstly, we study the existence and local stability of positive equilibrium of the new system. And, by choosing the time delay $\tau$ as a bifurcation parameter, we show that Hopf bifurcation may occur as the time delay $\tau$ passes through some critical values. Secondly, by use of normal form theory and central manifold argument, we establish the direction and stability of Hopf bifurcation. At last, some simple discussion is presented.

\section{Introduction}

Since the pioneering theoretical works by Lotka [1] and Volterra [2], there were a lot of authors who studied all kinds of predator-prey models modeled by ordinary differential equations (ODEs) [3-5]. To reflect that the dynamical behavior of the models depends on the past history of the system, it is often necessary to incorporate time delays into the models. Therefore, a more realistic predator-prey model should be described by delayed differential equations (DDEs) [6-26]. Some of them investigated discrete delays [6-20]; others were about distributed delays [21-24]; and both discrete and distributed delays were studied in [25]. In general, delay differential equations exhibit more complicated dynamics on stability, periodic structure, bifurcation, and so on [26].

In the natural world, many individuals have a life story that takes them through two stages, immature and mature. The predator only catches the mature prey, as the immature preys are protected by their eggshells or refuge. Some predator-prey models with stage structure were investigated in [27-33]. Motivated by $[25,27,31]$ and the references cited therein, in the present paper, we will consider the following stage-structured predator-prey model with discrete and distributed delay:

$$
\begin{aligned}
& x_{1}^{\prime}(t)=r x_{2}(t)-d_{1} x_{1}(t)-r e^{-d_{1} \tau} x_{2}(t-\tau), \\
& x_{2}^{\prime}(t)=r e^{-d_{1} \tau} x_{2}(t-\tau)-d_{2} x_{2}(t)-b_{1} x_{2}(t) y(t), \\
& y^{\prime}(t)=y(t)\left[-d_{3}+b_{2} \int_{-\infty}^{t} F(t-s) x_{2}(s) \mathrm{d} s-\alpha y(t)\right],
\end{aligned}
$$

where $x_{1}(t), x_{2}(t)$, and $y(t)$ can be interpreted as the population densities of the immature prey, mature prey, and predator at time $t$, respectively. $r$ denotes the birth rate of the prey population; $d_{1}, d_{2}$, and $d_{3}$ denote the death rate of the immature prey, mature prey, and the predator; $\alpha$ is the density-depended death rate of the predator; $b_{1}$ denotes the per capita per unit time predation rate of the predator; the term $b_{2} \int_{-\infty}^{t} F(t-s) x_{2}(s) \mathrm{d} s$ is the conversion rate from prey to predator, and the distributed delay may interpret as digest delay. The function $F(s)$ is called the delayed kernel that is a nonnegative bounded function defined on $[0, \infty)$. Following 
the ideas of Cushing et al. [34], we define $F(t)$ as the following weak kernel function:

$$
F(t)=\beta e^{-\beta t}, \quad \beta>0 .
$$

Next, we define a new variable:

$$
u(t)=\int_{-\infty}^{t} \beta e^{-\beta(t-s)} x_{2}(s) \mathrm{d} s .
$$

Then by use of linear chain trick technique, system (1) can be transformed into the following equivalent system:

$$
\begin{aligned}
& x_{1}^{\prime}(t)=r x_{2}(t)-d_{1} x_{1}(t)-r e^{-d_{1} \tau} x_{2}(t-\tau), \\
& x_{2}^{\prime}(t)=r e^{-d_{1} \tau} x_{2}(t-\tau)-d_{2} x_{2}(t)-b_{1} x_{2}(t) y(t), \\
& u^{\prime}(t)=\beta x_{2}(t)-\beta u(t), \\
& y^{\prime}(t)=y(t)\left[-d_{3}+b_{2} u(t)-\alpha y(t)\right] .
\end{aligned}
$$

The organization of this paper is as follows: In Section 2, we will get the conditions for the existence and stability of positive equilibrium of system (4). The occurring condition for Hopf bifurcation is also obtained. In Section 3, by use of normal form theory and central manifold argument, we illustrate the direction and stability of Hopf bifurcation. In Section 4, we give some brief discussion.

\section{Stability of Positive Equilibrium and Existence of Hopf Bifurcation}

In this section, we will firstly investigate the existence and stability of positive equilibrium of system (4) then study the effect of time delay on the system (4); that is, we will choose $\tau$ as bifurcating parameter to analyze Hopf bifurcation.

Theorem 1. There exists a unique positive equilibrium $E^{*}$ for system (4), if assumption

(H1) $r e^{-d_{2} \tau}-d_{2}>0$ holds. And $E^{*}=\left(x_{1}^{*}, x_{2}^{*}, u^{*}, y^{*}\right)$, with

$$
\begin{aligned}
& x_{1}^{*}=\frac{r\left(1-e^{-d_{1} \tau}\right)\left(\alpha r e^{-d_{1} \tau}-\alpha d_{2}+b_{1} b_{3}\right)}{b_{1} b_{2} d_{1}}, \\
& x_{2}^{*}=u^{*}=\frac{\alpha\left(r e^{-d_{1} \tau}-d_{2}\right)+b_{1} b_{3}}{b_{1} b_{2}}, \\
& y^{*}=\frac{r e^{-d_{1} \tau}-d_{2}}{b_{1}} .
\end{aligned}
$$

Linearizing system (4) at $E^{*}$, we get

$$
\begin{aligned}
& x_{1}^{\prime}(t)=-d_{1} x_{1}(t)+r x_{2}(t)-r e^{-d_{1} \tau} x_{2}(t-\tau), \\
& x_{2}^{\prime}(t)=\left(-d_{2}-b_{1} y^{*}\right) x_{2}(t)+r e^{-d_{1} \tau} x_{2}(t-\tau)-b_{1} x_{2}^{*} y(t), \\
& u^{\prime}(t)=\beta x_{2}(t)-\beta u(t), \\
& y^{\prime}(t)=b_{2} y^{*} u(t)-\alpha y^{*} y(t),
\end{aligned}
$$

and the characteristic equation for system (6) takes the form

$$
\begin{aligned}
\lambda^{4} & +h_{1} \lambda^{3}+h_{2} \lambda^{2}+h_{3} \lambda+h_{4} \\
& +\left[h_{5} \lambda^{3}+h_{6} \lambda^{2}+h_{7} \lambda+h_{8}\right] e^{-\lambda \tau}=0,
\end{aligned}
$$

where

$$
\begin{aligned}
& h_{1}=\left(d_{1}+d_{2}+b_{1} y^{*}\right)+\left(\beta+\alpha y^{*}\right), \\
& h_{2}=d_{1}\left(d_{2}+b_{1} y^{*}\right)+\left(\beta+\alpha y^{*}\right)\left(d_{1}+d_{2}+b_{1} y^{*}\right)+\alpha \beta y^{*}, \\
& h_{3}=d_{1}\left(d_{2}+b_{1} y^{*}\right)\left(\beta+\alpha y^{*}\right)+\alpha \beta y^{*}\left(d_{1}+d_{2}+b_{1} y^{*}\right), \\
& h_{4}=\alpha \beta d_{1} y^{*}\left(d_{2}+b_{1} y^{*}\right)+\beta b_{1} b_{2} x_{2}^{*} y^{*}, \\
& h_{5}=-r e^{-d_{1} \tau} \\
& h_{6}=-\left(\beta+\alpha y^{*}+d_{1}\right) r e^{-d_{1} \tau}, \\
& h_{7}=-\left(\alpha \beta y^{*}+d_{1} \beta+\alpha d_{1} y^{*}\right) r e^{-d_{1} \tau}, \\
& h_{8}=-\alpha \beta d_{1} y^{*} r e^{-d_{1} \tau} .
\end{aligned}
$$

Note that when $\tau=0$, (7) becomes

$\lambda^{4}+\left(h_{1}+h_{5}\right) \lambda^{3}+\left(h_{2}+h_{6}\right) \lambda^{2}+\left(h_{3}+h_{7}\right) \lambda+h_{4}+h_{8}=0$.

It is easy to confirm that

$$
\begin{gathered}
h_{1}+h_{5}=d_{1}+\beta+\alpha y^{*}>0, \\
h_{2}+h_{6}=\left(\beta+\alpha y^{*}\right) d_{1}+\alpha \beta y^{*}>0, \\
h_{3}+h_{7}=\alpha \beta y^{*} d_{1}>0, \\
h_{4}+h_{8}=\alpha \beta b_{1} b_{2} x_{2}^{*} y^{*}>0, \\
\left(h_{1}+h_{5}\right)\left(h_{2}+h_{6}\right)-\left(h_{3}+h_{7}\right)>0, \\
\left(h_{1}+h_{5}\right)\left[\left(h_{2}+h_{6}\right)\left(h_{3}+h_{7}\right)-\left(h_{1}+h_{5}\right)\left(h_{4}+h_{8}\right)\right] \\
-\left(h_{3}+h_{7}\right)^{2}>0 .
\end{gathered}
$$

Thus, by the Routh-Hurwitz criterion we know that all the roots of (9) have negative real parts, which means that the positive equilibrium $E^{*}$ is locally asymptotically stable for $\tau=$ 0 .

Next, we will consider the case for $\tau>0$. Suppose that there is a pure imaginary $\operatorname{root} \lambda=i \omega, \omega>0$. Then we get

$$
\begin{aligned}
\omega^{4}- & h_{1} i \omega^{3}-h_{2} \omega^{2}+h_{3} i \omega+h_{4} \\
& +\left(-h_{5} i \omega^{3}-h_{6} \omega^{2}+h_{7} i \omega+h_{8}\right)(\cos \omega \tau-i \sin \omega \tau)=0 .
\end{aligned}
$$

Separating the real and imaginary parts, we have

$\left(h_{6} \omega^{2}-h_{8}\right) \cos \omega \tau+\left(h_{5} \omega^{3}-h_{7} \omega\right) \sin \omega \tau=\omega^{4}-h_{2} \omega^{2}+h_{4}$,

$\left(h_{6} \omega^{2}-h_{8}\right) \sin \omega \tau+\left(-h_{5} \omega^{3}+h_{7} \omega\right) \cos \omega \tau=h_{1} \omega^{3}-h_{3} \omega$. 
Incorporating $\sin ^{2} \omega \tau+\cos ^{2} \omega \tau=1$, we have

$$
\omega^{8}+f_{1} \omega^{6}+f_{2} \omega^{4}+f_{3} \omega^{2}+f_{4}=0
$$

where

$$
\begin{aligned}
& f_{1}=h_{1}^{2}-2 h_{2}-h_{5}^{2}, \\
& f_{2}=2 h_{4}+h_{2}^{2}-2 h_{1} h_{3}-h_{6}^{2}+2 h_{5} h_{7}, \\
& f_{3}=h_{3}^{2}-2 h_{2} h_{4}+2 h_{6} h_{8}-h_{7}^{2}, \\
& f_{4}=h_{4}^{2}-h_{8}^{2} .
\end{aligned}
$$

Denote $z=\omega^{2}$. Then (13) becomes

$$
z^{4}+f_{1} z^{3}+f_{2} z^{2}+f_{3} z+f_{4}=0
$$

Let

$$
G(z)=z^{4}+f_{1} z^{3}+f_{2} z^{2}+f_{3} z+f_{4}
$$

Then the following assumption holds true.

(H2) Equation (15) has at least one positive real root.

In fact, if all the parameters of system (4) are given, it is easy to calculate the root of (15). Since $\lim _{z \rightarrow \infty} G(z)=+\infty$, we conclude that if $f_{4}<0$, then (15) has at least one positive real root. Without loss of generality, we assume that (15) has four positive root, defined by $z_{1}, z_{2}, z_{3}, z_{4}$, respectively. Then (13) has four positive roots as

$$
\begin{array}{ll}
\omega_{1}=\sqrt{z_{1}}, & \omega_{2}=\sqrt{z_{2}}, \\
\omega_{3}=\sqrt{z_{3}}, & \omega_{4}=\sqrt{z_{4}} .
\end{array}
$$

From (12), we obtain

$$
\begin{aligned}
\sin \omega \tau= & \left(h_{5} \omega^{7}+\left(h_{1} h_{6}-h_{7}-h_{2} h_{5}\right) \omega^{5}\right. \\
& +\left(h_{2} h_{7}+h_{4} h_{5}-h_{1} h_{8}-h_{3} h_{6}\right) \omega^{3} \\
& \left.+\left(h_{3} h_{8}-h_{4} h_{7}\right) \omega\right) \\
& \times\left(h_{5}^{2} \omega^{6}+\left(h_{6}^{2}-2 h_{5} h_{7}\right) \omega^{4}\right. \\
& \left.+\left(h_{7}^{2}-2 h_{6} h_{8}\right) \omega^{2}+h_{8}^{2}\right)^{-1} \\
\cos \omega \tau= & \left(\left(h_{6}-h_{1} h_{5}\right) \omega^{6}+\left(h_{1} h_{7}+h_{3} h_{5}-h_{2} h_{6}-h_{8}\right) \omega^{4}\right. \\
& \left.+\left(h_{4} h_{6}+h_{2} h_{8}-h_{3} h_{7}\right) \omega^{2}+h_{4} h_{8}\right) \\
\times & \left(h_{5}^{2} \omega^{6}+\left(h_{6}^{2}-2 h_{5} h_{7}\right) \omega^{4}\right. \\
& \left.\quad+\left(h_{7}^{2}-2 h_{6} h_{8}\right) \omega^{2}+h_{8}^{2}\right)^{-1} .
\end{aligned}
$$

\section{Denote}

$$
\begin{aligned}
& e_{1}=h_{5}^{2}, \quad e_{2}=h_{6}^{2}-2 h_{5} h_{7}, \\
& e_{3}=h_{7}^{2}-2 h_{6} h_{8}, \quad e_{4}=h_{8}^{2}, \\
& e_{5}=h_{5}, \quad e_{6}=h_{1} h_{6}-h_{7}-h_{2} h_{5}, \\
& e_{7}=h_{2} h_{7}+h_{4} h_{5}-h_{1} h_{8}-h_{3} h_{6}, \\
& e_{8}=h_{3} h_{8}-h_{4} h_{7}, \\
& e_{9}=h_{6}-h_{1} h_{5}, \\
& e_{10}=h_{1} h_{7}+h_{3} h_{5}-h_{2} h_{6}-h_{8}, \\
& e_{11}=h_{4} h_{6}+h_{2} h_{8}-h_{3} h_{7}, \quad e_{12}=-h_{4} h_{8} .
\end{aligned}
$$

Then $\cos \omega \tau$ can be written as

$$
\cos \omega \tau=\frac{e_{9} \omega^{6}+e_{10} \omega^{4}+e_{11} \omega^{2}+e_{12}}{e_{1} \omega^{6}+e_{2} \omega^{4}+e_{3} \omega^{2}+e_{4}},
$$

from which we can get

$$
\begin{array}{r}
\tau_{k}^{(j)}=\frac{1}{\omega_{k}}\left\{\arccos \left(\frac{e_{9} \omega_{k}^{6}+e_{10} \omega_{k}^{4}+e_{11} \omega_{k}^{2}+e_{12}}{e_{1} \omega_{k}^{6}+e_{2} \omega_{k}^{4}+e_{3} \omega_{k}^{2}+e_{4}}\right)+2 j \pi\right\}, \\
k=1,2,3,4 ; j=0,1,2, \ldots
\end{array}
$$

Thus, $\pm i \omega_{k}$ is a pair of purely imaginary root of (7). Define

$$
\tau_{0}=\tau_{k_{0}}^{(0)}=\min _{k \in\{1,2,3,4\}}\left\{\tau_{k}^{(0)}\right\}, \quad \omega_{0}=\omega,
$$

In order to obtain the main result, it is necessary to make the following assumption:

(H3) $\left.\operatorname{Re}(d \lambda / d \tau)\right|_{\tau=\tau_{0}} \neq 0$.

Taking the derivative of $\lambda$ with respect to $\tau$ in (7), it is easy to obtain

$$
\begin{aligned}
\left(4 \lambda^{3}\right. & \left.+3 h_{1} \lambda^{2}+2 h_{2} \lambda+h_{3}\right) \frac{d \lambda}{d \tau} \\
& +\left(3 h_{5} \lambda^{2}+2 h_{6} \lambda+h_{7}\right) e^{-\lambda \tau} \frac{d \lambda}{d \tau} \\
& -\left(h_{5} \lambda^{3}+h_{6} \lambda^{2}+h_{7} \lambda+h_{8}\right) e^{-\lambda \tau}\left(\lambda+\tau \frac{d \lambda}{d \tau}\right)=0,
\end{aligned}
$$

and it may be rewritten as

$$
\begin{aligned}
\frac{d \lambda}{d \tau}=\left(\left(h_{5} \lambda^{4}+\right.\right. & \left.\left.h_{6} \lambda^{3}+h_{7} \lambda^{2}+h_{8} \lambda\right) e^{-\lambda \tau}\right) \\
\times( & 4 \lambda^{3}+3 h_{1} \lambda^{2}+2 h_{2} \lambda+h_{3} \\
& +\left(3 h_{5} \lambda^{2}+2 h_{6} \lambda+h_{7}\right) e^{-\lambda \tau} \\
& \left.-\left(h_{5} \lambda^{3}+h_{6} \lambda^{2}+h_{7} \lambda+h_{8}\right) \tau e^{-\lambda \tau}\right)^{-1},
\end{aligned}
$$


or equivalently, we have

$$
\begin{aligned}
\left(\frac{d \lambda}{d \tau}\right)^{-1}= & 4 \lambda^{3}+3 h_{1} \lambda^{2}+2 h_{2} \lambda+h_{3} \\
& +\left(3 h_{5} \lambda^{2}+2 h_{6} \lambda+h_{7}\right) e^{-\lambda \tau} \\
& \left.-\left(h_{5} \lambda^{3}+h_{6} \lambda^{2}+h_{7} \lambda+h_{8}\right) \tau e^{-\lambda \tau}\right) \\
& \times\left(\left(h_{5} \lambda^{4}+h_{6} \lambda^{3}+h_{7} \lambda^{2}+h_{8} \lambda\right) e^{-\lambda \tau}\right)^{-1} \\
= & \left(4 \lambda^{3}+3 h_{1} \lambda^{2}+2 h_{2} \lambda+h_{3}\right. \\
& \left.+\left(3 h_{5} \lambda^{2}+2 h_{6} \lambda+h_{7}\right) e^{-\lambda \tau}\right) \\
& \times\left(h_{5} \lambda^{4}+h_{6} \lambda^{3}+h_{7} \lambda^{2}+h_{8} \lambda\right)^{-1}-\frac{\tau}{\lambda}
\end{aligned}
$$

Taking $\lambda=i \omega$ into the above equation, we get

$$
\begin{aligned}
\left(\frac{d \lambda}{d \tau}\right)^{-1} & \left(-4 i \omega^{3}-3 h_{1} \omega^{2}+2 h_{2} i \omega+h_{3}\right. \\
& \left.+\left(-3 h_{5} \omega^{2}+2 h_{6} i \omega+h_{7}\right)(\cos \omega \tau-i \sin \omega \tau)\right) \\
& \times\left(h_{5} \omega^{4}-h_{6} i \omega^{3}-h_{7} \omega^{2}+h_{8} i \omega\right)^{-1}-\frac{\tau}{i \omega} \\
= & \left.-3 h_{1} \omega^{2}+h_{3}-\left(3 h_{5} \omega^{2}-h_{7}\right) \cos \omega \tau+2 h_{6} \sin \omega \tau\right) \\
& \times\left(h_{5} \omega^{4}-h_{7} \omega^{2}+i\left(h_{8} \omega-h_{6} \omega^{3}\right)\right)^{-1} \\
+ & \left(i \left[-4 \omega^{3}+2 h_{2} \omega+2 h_{6} \omega \cos \omega \tau\right.\right. \\
& \left.\left.+\left(3 h_{5} \omega^{2}-h_{7}\right) \sin \omega \tau\right]\right) \\
\times & \left(h_{5} \omega^{4}-h_{7} \omega^{2}+i\left(h_{8} \omega-h_{6} \omega^{3}\right)\right)^{-1}-\frac{\tau}{i \omega} .
\end{aligned}
$$

Denote

$$
Q=\left(h_{5} \omega^{4}-h_{7} \omega^{2}\right)^{2}+\left(h_{8} \omega-h_{6} \omega^{3}\right)^{2}>0 .
$$

Then we have

$$
\begin{aligned}
& Q \operatorname{Re}\left(\frac{d \lambda}{d \tau}\right)^{-1} \\
& =\left[-3 h_{1} \omega^{2}+h_{3}-\left(3 h_{5} \omega^{2}-h_{7}\right) \cos \omega \tau+2 h_{6} \sin \omega \tau\right] \\
& \quad \times\left(h_{5} \omega^{4}-h_{7} \omega^{2}\right) \\
& \quad+\left[-4 \omega^{3}+2 h_{2} \omega+2 h_{6} \omega \cos \omega \tau+\left(3 h_{5} \omega^{2}-h_{7}\right) \sin \omega \tau\right] \\
& \quad \times\left(h_{8} \omega-h_{6} \omega^{3}\right) .
\end{aligned}
$$

Note that

$$
\operatorname{Sign}\left\{\left.\operatorname{Re}\left(\frac{d \lambda}{d \tau}\right)\right|_{\tau=\tau_{0}}\right\}=\operatorname{Sign}\left\{\left.\operatorname{Re}\left(\frac{d \lambda}{d \tau}\right)^{-1}\right|_{\tau=\tau_{0}}\right\}
$$

Now, we can employ a result from [35] to analyze (7).

Lemma 2 (see [35]). Consider the exponential polynomial

$$
\begin{aligned}
P( & \left.\lambda, e^{-\lambda \tau_{1}}, e^{-\lambda \tau_{2}}, \ldots, e^{-\lambda \tau_{m}}\right) \\
= & \lambda^{n}+p_{1}^{(0)} \lambda^{n-1}+\cdots+p_{n-1}^{(0)} \lambda+p_{n}^{(0)} \\
& +\left[p_{1}^{(1)} \lambda^{n-1}+\cdots+p_{n-1}^{(1)} \lambda+p_{n}^{(1)}\right] e^{-\lambda \tau_{1}} \\
& +\cdots+\left[p_{1}^{(m)} \lambda^{n-1}+\cdots+p_{n-1}^{(m)} \lambda+p_{n}^{(m)}\right] e^{-\lambda \tau_{m}},
\end{aligned}
$$

where $\tau_{i} \geq 0(i=1,2, \ldots, m)$ and $p_{j}^{(i)}(i=0,1, \ldots, m ; j=$ $1,2, \ldots, n)$ are constants. As $\left(\tau_{1}, \tau_{2}, \ldots, \tau_{m}\right)$ vary, the sum of the order of the zeros of $P\left(\lambda, e^{-\lambda \tau_{1}}, \ldots, e^{-\lambda \tau_{m}}\right)$ on the open right half-plane can change only if a zero appears on or crosses the imaginary axis.

From Lemma 2, it is easy to obtain the following theorem.

Theorem 3. Suppose that (H1), (H2), and (H3) hold. Then the following results hold true.

(i) The positive equilibrium $E^{*}$ of system (4) $\left(x_{1}^{*}, x_{2}^{*}\right.$, $\left.u^{*}, y^{*}\right)$ is asymptotically stable for $\tau \in\left[0, \tau_{0}\right)$.

(ii) The positive equilibrium $E^{*}$ of system (4) undergoes a Hopf bifurcation when $\tau=\tau_{0}$. That is system (4) has a periodic solution bifurcating from the positive equilibrium $E^{*}$ near $\tau=\tau_{0}$.

\section{Direction and Stability of the Hopf Bifurcation}

In this section, we will derive the explicit formulae for determining the properties of the Hopf bifurcation at critical value of $\tau_{0}$ by using the normal form and the center manifold theory [35]. Throughout this section, we always assume that system (4) undergoes Hopf bifurcation at the positive equilibrium $E^{*}$ for $\tau=\tau_{0}$, and then $\pm \omega_{0}$ is the corresponding purely imaginary roots of the characteristic equation at the positive equilibrium $E^{*}$.

Let $u_{1}=x_{1}-x_{1}^{*}, u_{2}=x_{2}-x_{2}^{*}, u_{3}=u-u^{*}, u_{4}=y-$ $y^{*}, \overline{u_{i}}(t)=u_{i}(\tau t)$, and $\tau=\tau_{0}+\mu$, and dropping the bars for simplification of notations, then system (4) is transformed into FDE defined in $C=C\left([-1,0], R^{4}\right)$ as

$$
u^{\prime}(t)=L_{\mu}\left(u_{t}\right)+g\left(\mu, x_{t}\right),
$$


where $u_{t}=\left(u_{1}(t), u_{2}(t), u_{3}(t), u_{4}(t)\right)^{T} \in R^{4}, L_{\mu}: C \rightarrow R, g$ : $R \times C \rightarrow R$, and

$$
\begin{aligned}
L_{\mu}(\phi) & \left(\tau_{0}+\mu\right)\left(\begin{array}{cccc}
-d_{1} & r & 0 & 0 \\
0 & -d_{2}-b_{1} y^{*} & 0 & -b_{1} x_{2}^{*} \\
0 & \beta & -\beta & 0 \\
0 & 0 & b_{2} y^{*} & -\alpha y^{*}
\end{array}\right) \\
& \times\left(\begin{array}{c}
\phi_{1}(0) \\
\phi_{2}(0) \\
\phi_{3}(0) \\
\phi_{4}(0)
\end{array}\right) \\
& +\left(\tau_{0}+\mu\right)\left(\begin{array}{cccc}
0 & -r e^{-d_{1} \tau} & 0 & 0 \\
0 & r e^{-d_{1} \tau} & 0 & 0 \\
0 & 0 & 0 & 0 \\
0 & 0 & 0 & 0
\end{array}\right)\left(\begin{array}{l}
\phi_{1}(-1) \\
\phi_{2}(-1) \\
\phi_{3}(-1) \\
\phi_{4}(-1)
\end{array}\right)
\end{aligned}
$$$$
g(\mu, \phi)=\left(\tau_{0}+\mu\right)\left(\begin{array}{c}
0 \\
-b_{1} \phi_{2}(0) \phi_{4}(0) \\
0 \\
b_{2} \phi_{4}(0) \phi_{3}(0)-\alpha \phi_{4}(0)^{2}
\end{array}\right),
$$

where $\phi(\theta)=\left(\phi_{1}(\theta), \phi_{2}(\theta), \phi_{3}(\theta), \phi_{4}(\theta)\right)^{T} \in C$. By the Riesz representation theorem, there exists a function $\eta(\theta, \mu)$ of bounded variation for $\theta \in[-1,0]$, such that

$$
L_{\mu} \phi=\int_{-1}^{0} \mathrm{~d} \eta(\theta, \mu) \phi(\theta), \quad \text { for } \phi \in C .
$$

In fact, we can choose

$$
\begin{aligned}
\eta(\theta, \mu) & =\left(\tau_{0}+\mu\right) \\
& \times\left(\begin{array}{cccc}
-d_{1} & r & 0 & 0 \\
0 & -d_{2}-b_{1} y^{*} & 0 & -b_{1} x_{2}^{*} \\
0 & \beta & -\beta & 0 \\
0 & 0 & b_{2} y^{*} & -\alpha y^{*}
\end{array}\right) \delta(\theta) \\
& +\left(\tau_{0}+\mu\right)\left(\begin{array}{cccc}
0 & -r e^{-d_{1}\left(\tau_{0}+\mu\right)} & 0 & 0 \\
0 & r e^{-d_{1}\left(\tau_{0}+\mu\right)} & 0 & 0 \\
0 & 0 & 0 & 0 \\
0 & 0 & 0 & 0
\end{array}\right) \delta(\theta+1),
\end{aligned}
$$

where $\delta$ is the Dirac delta function. For $\phi \in C^{1}\left([-1,0], R^{4}\right)$, define

$$
\begin{gathered}
A(\mu) \phi= \begin{cases}\frac{\mathrm{d} \phi(\theta)}{\mathrm{d} \theta}, & \theta \in[-1,0), \\
\int_{-1}^{0} \mathrm{~d} \eta(s, \mu) \phi(s), & \theta=0,\end{cases} \\
R(\mu) \phi= \begin{cases}0, & \theta \in[-1,0), \\
g(\mu, \phi), & \theta=0 .\end{cases}
\end{gathered}
$$

Then system (32) is equivalent to

$$
u^{\prime}(t)=A(\mu) u_{t}+R(\mu) u_{t},
$$

where $u_{t}=u(t+\theta), \theta \in[-1,0]$. For $\psi \in C^{1}\left([0,1], v\left(R^{4}\right)^{*}\right)$, define

$$
A^{*} \psi(s)= \begin{cases}-\frac{\mathrm{d} \psi(s)}{\mathrm{d} s}, & s \in(0,1], \\ \int_{-1}^{0} \mathrm{~d} \eta^{T}(t, 0) \psi(-t), & s=0\end{cases}
$$

and a bilinear inner product

$$
\begin{aligned}
\langle\psi(s), \phi(\theta)\rangle= & \bar{\psi}(0) \phi(0) \\
& -\int_{-1}^{0} \int_{\xi=0}^{\theta} \bar{\psi}(\xi-\theta) \mathrm{d} \eta(\theta) \phi(\xi) \mathrm{d} \xi,
\end{aligned}
$$

where $\eta(\theta)=\eta(\theta, 0)$. Then $A(0)$ and $A^{*}$ are adjoint operators. By the discussion in Section 2, we know that $\pm i \omega_{0} \tau_{0}$ are eigenvalues of $A(0)$. Thus, they are also eigenvalues of $A^{*}$. We need to compute the eigenvector of $A(0)$ and $A^{*}$ corresponding to $i \omega_{0} \tau_{0}$ and $-i \omega_{0} \tau_{0}$, respectively.

Suppose that $q(\theta)=\left(1, q_{1}, q_{2}, q_{3}\right)^{T} e^{i \theta \omega_{0} \tau_{0}}$ is the eigenvectors of $A(0)$ corresponding to $i \omega_{0} \tau_{0}$. Then $A(0) q(\theta)=$ $i \omega_{0} \tau_{0} q(\theta)$. It follows from the definition of $A(0)$ and $\eta(\theta, \mu)$ that

$$
\begin{gathered}
\tau_{0}\left(\begin{array}{cccc}
-d_{1} & r & 0 & 0 \\
0 & -d_{2}-b_{1} y^{*} & 0 & -b_{1} x_{2}^{*} \\
0 & \beta & -\beta & 0 \\
0 & 0 & b_{2} y^{*} & -\alpha y^{*}
\end{array}\right) q(0) \\
\quad+\tau_{0}\left(\begin{array}{cccc}
0 & -r e^{-d_{1} \tau_{0}} & 0 & 0 \\
0 & r e^{-d_{1} \tau_{0}} & 0 & 0 \\
0 & 0 & 0 & 0 \\
0 & 0 & 0 & 0
\end{array}\right) q(-1)=i \omega_{0} \tau_{0} q(0)
\end{gathered}
$$

Because of $q(-1)=q(0) e^{-i \omega_{0} \tau_{0}}$, then we get

$$
\begin{gathered}
\left(\begin{array}{cccc}
i \omega_{0}+d_{1} & -r+r e^{-d_{1} \tau_{0}} e^{-i \omega_{0} \tau_{0}} & 0 & 0 \\
0 & i \omega_{0}+d_{2}+b_{1} y^{*}-r e^{-d_{1} \tau_{0}} e^{-i \omega_{0} \tau_{0}} & 0 & b_{1} x_{2}^{*} \\
0 & -\beta & i \omega_{0}+\beta & 0 \\
0 & 0 & -b_{2} y^{*} & i \omega_{0}+\alpha y^{*}
\end{array}\right) \\
\times\left(\begin{array}{c}
1 \\
q_{1} \\
q_{2} \\
q_{3}
\end{array}\right)=\left(\begin{array}{l}
0 \\
0 \\
0 \\
0
\end{array}\right),
\end{gathered}
$$

from which we obtain

$$
\begin{aligned}
& q_{1}=\frac{i \omega_{0}-d_{1}}{r\left(1-e^{-d_{1} \tau_{0}} e^{-i \omega_{0} \tau_{0}}\right)}, \\
& q_{2}=\frac{\beta\left(d_{1}-i \omega_{0}\right)}{r\left(i \omega_{0}+\beta\right)\left(1-e^{-d_{1} \tau_{0}} e^{-i \omega_{0} \tau_{0}}\right)}, \\
& q_{3}=\frac{b_{2} y^{*} \beta\left(i \omega_{0}+d_{1}\right)}{r\left(i \omega_{0}+\beta\right)\left(i \omega_{0}+\alpha y^{*}\right)\left(1-e^{-d_{1} \tau_{0}} e^{-i \omega_{0} \tau_{0}}\right)} .
\end{aligned}
$$


Similarly, let $q^{*}(\theta)=D\left(1, q_{1}^{*}, q_{2}^{*}, q_{3}^{*}\right) e^{i \theta \omega_{0} \tau_{0}}$ be the eigenvectors of $A^{*}$ corresponding to $-i \omega_{0} \tau_{0}$, and according to the definition of $A^{*}$ we have

$$
\begin{aligned}
& \tau_{0}\left(\begin{array}{cccc}
-d_{1} & 0 & 0 & 0 \\
r & -d_{2}-b_{1} y^{*} & \beta & 0 \\
0 & 0 & -\beta & b_{2} y^{*} \\
0 & -b_{1} x_{2}^{*} & 0 & -\alpha y^{*}
\end{array}\right) q^{*}(0) \\
& \quad+\tau_{0}\left(\begin{array}{ccccc}
0 & 0 & 0 & 0 \\
-r e^{-d_{1} \tau_{0}} & r e^{-d_{1} \tau_{0}} & 0 & 0 \\
0 & 0 & 0 & 0 \\
0 & 0 & 0 & 0
\end{array}\right) q^{*}(-1) \\
& =-i \omega_{0} \tau_{0} q^{*}(0) .
\end{aligned}
$$

Note that $q^{*}(-1)=q^{*}(0) e^{i \omega_{0} \tau_{0}}$. Then we get

$$
\begin{gathered}
\left(\begin{array}{cccc}
-i \omega_{0}+d_{1} & 0 & 0 & 0 \\
r e^{-d_{1} \tau_{0}} e^{i \omega_{0} \tau_{0}}-r & d_{2}-i \omega_{0}+b_{1} y^{*}-r e^{-d_{1} \tau_{0}} e^{i \omega_{0} \tau_{0}} & -\beta & 0 \\
0 & 0 & \beta-i \omega_{0} & -b_{2} y^{*} \\
0 & b_{1} x_{2}^{*} & 0 & \alpha y^{*}-i \omega_{0}
\end{array}\right) \\
\times\left(\begin{array}{l}
1 \\
q_{1}^{*} \\
q_{2}^{*} \\
q_{3}^{*}
\end{array}\right)=\left(\begin{array}{l}
0 \\
0 \\
0 \\
0
\end{array}\right),
\end{gathered}
$$

from which we can obtain

$$
\begin{aligned}
& q_{1}^{*}=\left(\left(i \omega_{0}-\alpha y^{*}\right)\left(i \omega_{0}+\beta\right)\left(1-e^{-d_{1} \tau_{0}} e^{i \omega_{0} \tau_{0}}\right)\right) \\
& \times\left(\left(i \omega_{0}+\beta\right)\left(i \omega_{0}-\alpha y^{*}\right)\right. \\
& \times\left(-i \omega_{0}+d_{2}+b_{1} y^{*}-r e^{-d_{1} \tau_{0}} e^{-i \omega_{0} \tau_{0}}\right) \\
&\left.-\beta b_{1} x_{2}^{*} b_{2} y^{*}\right)^{-1}, \\
& q_{2}^{*}=\left(r b_{1} x_{2}^{*} b_{2} y^{*}\left(i \omega_{0}+\beta\right)\left(1-e^{-d_{1} \tau_{0}} e^{i \omega_{0} \tau_{0}}\right)\right) \\
& \times\left(\left(\omega_{0}^{2}+\beta^{2}\right)\left(i \omega_{0}-\alpha y^{*}\right)\right. \\
& \quad \times\left(-i \omega_{0}+d_{2}+b_{1} y^{*}-r e^{-d_{1} \tau_{0}} e^{-i \omega_{0} \tau_{0}}\right) \\
&\left.\quad-\left(\beta-i \omega_{0}\right) \beta b_{1} x_{2}^{*} b_{2} y^{*}\right)^{-1}, \\
& q_{3}^{*}=\left(r b_{1} x_{2}^{*}\left(i \omega_{0}+\beta\right)\left(1-e^{-d_{1} \tau_{0}} e^{i \omega_{0} \tau_{0}}\right)\right) \\
& \times\left(\left(i \omega_{0}+\beta\right)\left(i \omega_{0}-\alpha y^{*}\right)\right. \\
& \quad \times\left(-i \omega_{0}+d_{2}+b_{1} y^{*}-r e^{-d_{1} \tau_{0}} e^{-i \omega_{0} \tau_{0}}\right) \\
&\left.\quad-\beta b_{1} x_{2}^{*} b_{2} y^{*}\right)^{-1} .
\end{aligned}
$$

By (40), we get

$$
\begin{aligned}
& \left\langle q^{*}(s), q(\theta)\right\rangle \\
& =\bar{D}\left(1, \bar{q}_{1}^{*}, \bar{q}_{2}^{*}, \bar{q}_{3}^{*}\right)\left(1, q_{1}, q_{2}, q_{3}\right)^{T} \\
& \quad-\int_{-1}^{0} \int_{\xi=0}^{\theta} \bar{D}\left(1, \bar{q}_{1}^{*}, \bar{q}_{2}^{*}, \bar{q}_{3}^{*}\right) \\
& \quad \times e^{-i \omega_{0} \tau_{0}(\xi-\theta)} \mathrm{d} \eta(\theta)\left(1, q_{1}, q_{2}, q_{3}\right)^{T} e^{i \omega_{0} \tau_{0} \xi} \mathrm{d} \xi
\end{aligned}
$$

$$
\begin{aligned}
& =\bar{D}\left\{1+q_{1} \bar{q}_{1}^{*}+q_{2} \bar{q}_{2}^{*}+q_{3} \bar{q}_{3}^{*}\right. \\
& \left.\quad-\int_{-1}^{0}\left(1, \bar{q}_{1}^{*}, \bar{q}_{2}^{*}, \bar{q}_{3}^{*}\right) \theta e^{i \omega_{0} \tau_{0} \theta} d \eta(\theta)\left(1, q_{1}, q_{2}, q_{3}\right)^{T}\right\} \\
& =\bar{D}\left[1+q_{1} \bar{q}_{1}^{*}+q_{2} \bar{q}_{2}^{*}+q_{3} \bar{q}_{3}^{*}\right. \\
& \left.\quad+\tau_{0} r q_{1}\left(\bar{q}_{1}^{*}-1\right) e^{-d_{2} \tau_{0}} e^{-i \omega_{0} \tau_{0}}\right] .
\end{aligned}
$$

Then we can choose $\bar{D}$ such that $\left\langle q^{*}(s), q(\theta)\right\rangle=1,\left\langle q^{*}(s)\right.$, $\bar{q}(\theta)\rangle=0$.

Nest, we will use the ideas in [35] to compute the coordinates describing center manifold $C_{0}$ at $\mu=0$. Define

$$
\begin{aligned}
z(t) & =\left\langle q^{*}, u_{t}\right\rangle, \\
W(t, \theta) & =u_{t}(\theta)-2 \operatorname{Re}\{z(t), q(\theta)\} .
\end{aligned}
$$

On the center manifold $C_{0}$, we have $W(t, \theta)=W(z(t)$, $\bar{z}(t), \theta)$, and

$$
W(z(t), \bar{z}(t), \theta)=W_{20}(\theta) \frac{z^{2}}{2}+W_{11}(\theta) z \bar{z}+W_{02} \frac{\bar{z}^{2}}{2}+\ldots,
$$

where $z$ and $\bar{z}$ are local coordinates for center manifold $C_{0}$ in the direction of $q^{*}(s)$ and $\bar{q}(\theta)$. Note that $W$ is real if $u_{t}$ is real, and we only consider real solutions. For the solution $u_{t} \in C_{0}$ of (38), since $\mu=0$ and (38), we have

$$
\begin{aligned}
z^{\prime}(t) & =i \omega_{0} \tau_{0} z+\bar{q}^{*}(0) g(0, W(z, \bar{z}, 0))+2 \operatorname{Re}\{z q(0)\} \\
& =i \omega_{0} \tau_{0} z+\bar{q}^{*}(0) g_{0}(z, \bar{z}) .
\end{aligned}
$$

Then, the above equation can be denoted as

$$
z^{\prime}(t)=i \omega_{0} \tau_{0} z(t)+f(z, \bar{z})
$$

where

$$
\begin{aligned}
f(z, \bar{z})= & \bar{q}^{*}(0) g_{0}(z, \bar{z})=f_{20} \frac{z^{2}}{2}+f_{11} z \bar{z} \\
& +f_{02} \frac{\bar{z}^{2}}{2}+f_{21} \frac{z^{2} \bar{z}}{2}+\cdots .
\end{aligned}
$$

From (48) and (49), we have

$$
\begin{aligned}
u_{t} & =\left(u_{1 t}(\theta), u_{2 t}(\theta), u_{3 t}(\theta), u_{4 t}(\theta)\right) \\
& =W(t, \theta)+2 \operatorname{Re}\{z(t), q(\theta)\} \\
& =W(t, \theta)+z q(\theta)+\bar{z} \overline{q(\theta)},
\end{aligned}
$$


and then we can obtain

$$
\begin{aligned}
u_{2 t}(0)= & W_{20}^{(2)}(0) \frac{z^{2}}{2}+W_{11}^{(2)}(0) z \bar{z} \\
& +W_{02}^{(2)}(0) \frac{\bar{z}^{2}}{2}+q_{1} z+\overline{q_{1}} \bar{z}+O\left(\left|z, \bar{z}^{3}\right|\right), \\
u_{3 t}(0)= & W_{20}^{(3)}(0) \frac{z^{2}}{2}+W_{11}^{(3)}(0) z \bar{z} \\
& +W_{02}^{(3)}(0) \frac{\bar{z}^{2}}{2}+q_{2} z+\overline{q_{2}} \bar{z}+O\left(|z, \bar{z}|^{3}\right), \\
u_{4 t}(0)= & W_{20}^{(4)}(0) \frac{z^{2}}{2}+W_{11}^{(4)}(0) z \bar{z} \\
& +W_{02}^{(4)}(0) \frac{\bar{z}^{2}}{2}+q_{3} z+\overline{q_{3}} \bar{z}+O\left(|(z, \bar{z})|^{3}\right) .
\end{aligned}
$$

From the definition of $g\left(\mu, u_{t}\right)$, we have

$$
\begin{aligned}
& f(z, \bar{z})=\bar{q}^{*}(0) g_{0}(z, \bar{z}) \\
& =\tau_{0} \bar{D}\left(1, \bar{q}_{1}^{*}, \bar{q}_{2}^{*}, \bar{q}_{3}^{*}\right) \\
& \times\left(\begin{array}{c}
0 \\
-2 b_{1} u_{2 t}(0) u_{4 t}(0) \\
0 \\
b_{2} u_{3 t}(0) u_{4 t}(0)-\alpha u_{4 t}^{2}(0)
\end{array}\right) \\
& =\tau_{0} \bar{D}\left\{z^{2}\left[-2 b_{1} \bar{q}_{1}^{*} q_{1} q_{3}+b_{2} \bar{q}_{3}^{*} q_{2} q_{3}-\alpha \bar{q}_{3}^{*} q_{3}^{2}\right]\right. \\
& +2 z \bar{z}\left[-2 b_{1} \bar{q}_{1}^{*} \operatorname{Re}\left\{q_{1} \overline{q_{3}}\right\}+b_{2} \bar{q}_{3}^{*} \operatorname{Re}\left\{q_{2} \overline{q_{3}}\right\}\right. \\
& \left.-\alpha \bar{q}_{3}^{*} \operatorname{Re}\left\{q_{3} \overline{q_{3}}\right\}\right] \\
& +\overline{z^{2}}\left[-b_{1} \bar{q}_{1}^{*} \overline{q_{1} q_{3}}+b_{2} \bar{q}_{3}^{*} \overline{q_{2} q_{3}}-\alpha \bar{q}_{3}^{*} \overline{q_{3} q_{3}}\right] \\
& +\frac{1}{2} z^{2} \bar{z}\left[-b_{1} q_{1}^{*}\left(W_{20}^{(2)}(0) \overline{q_{3}}+2 W_{11}^{(2)}(0) q_{3}\right.\right. \\
& \left.+2 W_{11}^{(4)}(0) q_{1}+W_{20}^{(4)}(0) \overline{q_{1}}\right) \\
& +b_{2} q_{3}^{*}\left(W_{20}^{(3)}(0) \overline{q_{3}}+2 W_{11}^{(3)}(0) q_{3}\right. \\
& \left.+2 W_{11}^{(4)}(0) q_{2}+W_{20}^{(4)}(0) \overline{q_{2}}\right) \\
& \left.-\alpha q_{3}^{*}\left(2 W_{20}^{(4)}(0) \overline{q_{3}}+4 W_{11}^{(4)}(0) q_{3}\right)\right] \\
& +\cdots\} \text {. }
\end{aligned}
$$

Comparing the coefficients with those of (52), we obtain

$$
\begin{aligned}
& f_{20}=2 \tau_{0} \bar{D} {\left[-b_{1} \bar{q}_{1}^{*} q_{1} q_{3}+b_{2} \bar{q}_{3}^{*} q_{2} q_{3}-\alpha \bar{q}_{3}^{*} q_{3}^{2}\right], } \\
& f_{11}=2 \tau_{0} \bar{D}\left[-b_{1} \bar{q}_{1}^{*} \operatorname{Re}\left\{q_{1} \overline{q_{3}}\right\}\right. \\
&\left.\quad+b_{2} \bar{q}_{3}^{*} \operatorname{Re}\left\{q_{2} \overline{q_{3}}\right\}-\alpha \bar{q}_{3}^{*} \operatorname{Re}\left\{q_{3} \overline{\bar{q}_{3}}\right\}\right], \\
& f_{02}=2 \tau_{0} \bar{D}\left[-b_{1} \bar{q}_{1}^{*} \overline{q_{1}} \overline{q_{3}}+b_{2} \bar{q}_{3}^{*} \overline{q_{2}} \overline{q_{3}}-\alpha \bar{q}_{3}^{*} \overline{q_{3}^{2}}\right],
\end{aligned}
$$

$$
\begin{aligned}
f_{21}=\tau_{0} \bar{D}\left[-b_{1} q_{1}^{*}(\right. & W_{20}^{(2)}(0) \overline{q_{3}} \\
& +2 W_{11}^{(2)}(0) q_{3}+2 W_{11}^{(4)}(0) q_{1} \\
& \left.+W_{20}^{(4)}(0) \overline{q_{1}}\right) \\
+b_{2} q_{3}^{*} & \left(W_{20}^{(3)}(0) \overline{q_{3}}\right. \\
& +2 W_{11}^{(3)}(0) q_{3}+2 W_{11}^{(4)}(0) q_{2} \\
& \left.+W_{20}^{(4)}(0) \overline{q_{2}}\right) \\
-\alpha q_{3}^{*} & \left.\left(2 W_{20}^{(4)}(0) \overline{q_{3}}+4 W_{11}^{(4)}(0) q_{3}\right)\right] .
\end{aligned}
$$

In order to determine $f_{21}$ we need to compute $W_{20}(\theta)$ and $W_{11}(\theta)$. From (38) and (48), we have

$$
\begin{aligned}
\dot{W} & =\dot{u}_{t}-\dot{z} q-\dot{\overline{z q}} \\
& = \begin{cases}A(0) W-2 \operatorname{Re}\left\{\bar{q}^{*}(0) g_{0} q(\theta)\right\}, & \theta \in[-1,0), \\
A(0) W-2 \operatorname{Re}\left\{\bar{q}^{*}(0) g_{0} q(\theta)\right\}+g_{0}, & \theta=0,\end{cases} \\
& =A(0) W-H(z, \bar{z}, \theta),
\end{aligned}
$$

where

$$
H(z, \bar{z}, \theta)=H_{20}(\theta) \frac{z^{2}}{2}+H_{11}(\theta) z \bar{z}+H_{02} \frac{\overline{z^{2}}}{2}+\cdots .
$$

Note that on the center manifold $C_{0}$ near the origin

$$
\dot{W}=W_{z} \dot{z}+W_{\bar{z}} \dot{\bar{z}},
$$

and thus we obtain

$$
\begin{aligned}
\left(A(0)-2 i \omega_{0} \tau_{0}\right) W_{20}(\theta) & =-H_{20}(\theta), \\
A(0) W_{11}(\theta) & =-H_{11}(\theta) .
\end{aligned}
$$

By (58), we know that, for $\theta \in[-1,0)$,

$H(z, \bar{z}, \theta)=-\bar{q}^{*}(0) g_{0} q(\theta)-q_{0}^{*} \overline{g_{0} q}(\theta)=-f q(\theta)-\bar{f} \overline{q(\theta)}$.

Comparing the coefficients with those in (59), we get

$$
\begin{aligned}
& H_{20}(\theta)=-f_{20} q(\theta)-\bar{f}_{02} \bar{q}(\theta), \\
& H_{11}(\theta)=-f_{11} q(\theta)-\bar{f}_{11} \bar{q}(\theta) .
\end{aligned}
$$

From (61), (63), and the definition of $A$, we have

$$
\dot{W}_{20}(\theta)=2 i \omega_{0} \tau_{0} W_{20}(\theta)+f_{20} q(\theta)+\bar{f}_{02} \bar{q}(\theta) .
$$

Noting that $q(\theta)=q(0) e^{i \omega_{0} \tau_{0} \theta}$, we get

$$
\begin{aligned}
W_{20}(\theta)= & \frac{i f_{20}}{\omega_{0} \tau_{0}} q(0) e^{i \omega_{0} \tau_{0} \theta} \\
& +\frac{i \bar{f}_{02}}{3 \omega_{0} \tau_{0}} \bar{q}(0) e^{-i \omega_{0} \tau_{0} \theta}+E_{1} e^{2 i \omega_{0} \tau_{0} \theta},
\end{aligned}
$$

where $E_{1}=\left(E_{1}^{(1)}, E_{1}^{(2)}, E_{1}^{(3)}, E_{1}^{(4)}\right) \in R^{4}$ is a constant vector. 
Similarly, from (61) and (62), we can get

$$
W_{11}(\theta)=\frac{i \bar{f}_{11}}{\omega_{0} \tau_{0}} \bar{q}(0) e^{-i \omega_{0} \tau_{0} \theta}+E_{2},
$$

where $E_{2}=\left(E_{2}^{(1)}, E_{2}^{(2)}, E_{2}^{(3)}, E_{2}^{(4)}\right) \in R^{4}$ is a constant vector.

Next, we will find out $E_{1}$ and $E_{2}$. In fact, from the definition of $A$ and (61), we can obtain

$$
\begin{gathered}
\int_{-1}^{0} \mathrm{~d} \eta(\theta) W_{20}(\theta)=2 i \omega_{0} \tau_{0} W_{20}(0)-H_{20}(0), \\
\int_{-1}^{0} \mathrm{~d} \eta(\theta) W_{11}(\theta)=-H_{11}(0),
\end{gathered}
$$

where $\eta(\theta)=\eta(0, \theta)$. From (59) and (61), when $\theta=0$ we have

$$
\begin{aligned}
H(z, \bar{z}, 0) & =-2 \operatorname{Re}\left\{\bar{q}^{*}(0) g_{0} q(0)\right\}+g_{0} \\
& =-\bar{q}^{*}(0) g_{0} q(0)-q^{*}(0) \overline{g_{0}} \bar{q}(0)+g_{0} \\
& =-g(z, \bar{z}) q(0)-\bar{g}(z, \bar{z}) \bar{q}(0)+g_{0} .
\end{aligned}
$$

That is,

$$
\begin{aligned}
H_{20}(0) & \frac{z^{2}}{2}+H_{11}(0) z \bar{z}+H_{02}(0) \frac{\overline{z^{2}}}{2} \\
= & -q(0)\left(f_{20} \frac{z^{2}}{2}+f_{11} z \bar{z}+f_{02} \frac{\overline{z^{2}}}{2}+\cdots\right) \\
& -\bar{q}(0)\left(\bar{f}_{20} \frac{\bar{z}^{2}}{2}+\bar{f}_{11} z \bar{z}+\bar{f}_{02} \frac{z^{2}}{2}+\cdots\right)+g_{0} .
\end{aligned}
$$

By (34), we have

$$
g_{0}=\tau_{0}\left(\begin{array}{c}
0 \\
-b_{1} u_{2 t}(0) u_{4 t}(0) \\
0 \\
b_{2} u_{3 t}(0) u_{4 t}(0)-\alpha u_{4 t}^{2}(0)
\end{array}\right)
$$

By (48) and (49), we have

$$
\begin{aligned}
u_{t}(\theta)= & W(t, \theta)+2 \operatorname{Re}\{z(t) q(\theta)\} \\
= & W(t, \theta)+z(t) q(\theta)+\bar{z}(t) \bar{q}(\theta) \\
= & W_{20}(\theta) \frac{z^{2}}{2}+W_{11}(\theta) z \bar{z}+z(t) q(\theta) \\
& +\bar{z}(t) \bar{q}(\theta)+\cdots .
\end{aligned}
$$

Thus, we obtain

$$
\begin{aligned}
H_{20}(0)=-f_{20} q(0)-\bar{f}_{02} \bar{q}(0)+2 \tau_{0}\left(\begin{array}{c}
0 \\
-b_{1} q_{1} q_{3} \\
0 \\
b_{2} q_{2} q_{3}-\alpha q_{3}^{2}
\end{array}\right), \\
H_{11}(0)=-f_{11} q(0)-\bar{f}_{11} \bar{q}(0)+2 \tau_{0} \\
\\
\qquad\left(\begin{array}{c}
-b_{1} \operatorname{Re}\left\{q_{1} \overline{q_{3}}\right\} \\
0 \\
b_{2} \operatorname{Re}\left\{q_{2} \overline{q_{3}}\right\}-\alpha \bar{q}_{3}^{*}\left|q_{3}\right|^{2}
\end{array}\right)
\end{aligned}
$$

Note that

$$
\begin{gathered}
\left(i \omega_{0} \tau_{0} I-\int_{-1}^{0} e^{i \omega_{0} \tau_{0} \theta} d \eta(\theta)\right) q(0)=0, \\
\left(-i \omega_{0} \tau_{0} I-\int_{-1}^{0} e^{-i \omega_{0} \tau_{0} \theta} d \eta(\theta)\right) \bar{q}(0)=0 .
\end{gathered}
$$

Then, by substituting (65) and (73) into (67), we will get

$$
\left(2 i \omega_{0} \tau_{0} I-\int_{-1}^{0} e^{2 i \omega_{0} \tau_{0} \theta} d \eta(\theta)\right) E_{1}=2 \tau_{0}\left(\begin{array}{c}
0 \\
-b_{1} q_{1} q_{3} \\
0 \\
b_{2} q_{2} q_{3}-\alpha q_{3}^{2}
\end{array}\right),
$$

or equivalently,

$$
\begin{aligned}
& \left(\begin{array}{cccc}
2 i \omega_{0}+d_{1} & -r+r e^{-d_{1} \tau_{0}} e^{-2 i \omega_{0} \tau_{0}} & 0 & 0 \\
0 & 2 i \omega_{0}+d_{2}+b_{1} y^{*}-r e^{-d_{1} \tau_{0}} e^{-2 i \omega_{0} \tau_{0}} & 0 & b_{1} x_{2}^{*} \\
0 & -\beta & 2 i \omega_{0}+\beta & 0 \\
0 & 0 & -b_{2} y^{*} & 2 i \omega_{0}-\alpha y^{*}
\end{array}\right) E_{1} \\
& =2\left(\begin{array}{c}
0 \\
-b_{1} q_{1} q_{3} \\
0 \\
b_{2} q_{2} q_{3}-\alpha q_{3}^{2}
\end{array}\right),
\end{aligned}
$$

from which we can get

$E_{1}$

$$
\begin{aligned}
& =2\left(\begin{array}{cccc}
2 i \omega_{0}+d_{1} & -r+r e^{-d_{1} \tau_{0}} e^{-2 i \omega_{0} \tau_{0}} & 0 & 0 \\
0 & 2 i \omega_{0}+d_{2}+b_{1} y^{*}-r e^{-d_{1} \tau_{0}} e^{-2 i \omega_{0} \tau_{0}} & 0 & b_{1} x_{2}^{*} \\
0 & -\beta & 2 i \omega_{0}+\beta & 0 \\
0 & 0 & -b_{2} y^{*} & 2 i \omega_{0}-\alpha y^{*}
\end{array}\right)^{-1} \\
& \times\left(\begin{array}{c}
0 \\
-b_{1} q_{1} q_{3} \\
0 \\
b_{2} q_{2} q_{3}-\alpha q_{3}^{2}
\end{array}\right) .
\end{aligned}
$$

Similarly, by substituting (66) and (74) into (68), we will get

$$
\begin{aligned}
& \left(\begin{array}{cccc}
d_{1} & -r+r e^{-d_{1} \tau_{0}} & 0 & 0 \\
0 & d_{2}+b_{1} y^{*}-r e^{-d_{1} \tau_{0}} & 0 & b_{1} x_{2}^{*} \\
0 & -\beta & \beta & 0 \\
0 & 0 & -b_{2} y^{*} & -\alpha y^{*}
\end{array}\right) E_{2} \\
& =2\left(\begin{array}{c}
0 \\
-b_{1} \operatorname{Re}\left\{q_{1} \overline{q_{3}}\right\} \\
0 \\
b_{2} \operatorname{Re}\left\{q_{2} \overline{q_{3}}\right\}-\alpha \operatorname{Re}\left\{q_{3} \overline{q_{3}}\right\}
\end{array}\right), \\
& E_{2}=2\left(\begin{array}{cccc}
d_{1} & -r+r e^{-d_{1} \tau_{0}} & 0 & 0 \\
0 & d_{2}+b_{1} y^{*}-r e^{-d_{1} \tau_{0}} & 0 & b_{1} x_{2}^{*} \\
0 & -\beta & \beta & 0 \\
0 & 0 & -b_{2} y^{*} & -\alpha y^{*}
\end{array}\right)^{-1} \\
& \times\left(\begin{array}{c}
0 \\
-b_{1} \operatorname{Re}\left\{q_{1} \overline{q_{3}}\right\} \\
0 \\
b_{2} \operatorname{Re}\left\{q_{2} \overline{q_{3}}\right\}-\alpha \operatorname{Re}\left\{q_{3} \overline{q_{3}}\right\}
\end{array}\right)
\end{aligned}
$$


Thus, we can determine $W_{20}(\theta)$ and $W_{11}(\theta)$ from (65) and (66). Furthermore, $f_{21}$ can be expressed by the parameters and delay. Thus, we can compute the following values:

$$
\begin{aligned}
c_{1}(0) & =\frac{i}{2 \omega_{0} \tau_{0}}\left(f_{20} f_{11}-2\left|f_{11}\right|^{2}-\frac{\left|f_{02}\right|^{2}}{3}\right)+\frac{f_{21}}{2}, \mu_{2} \\
& =-\frac{\operatorname{Re}\left\{c_{1}(0)\right\}}{\operatorname{Re}\left\{\lambda^{\prime}\left(\tau_{0}\right)\right\}}, \beta_{2} \\
& =2 \operatorname{Re}\left\{c_{1}(0)\right\}, T_{2} \\
& =-\frac{\operatorname{Im}\left\{c_{1}(0)\right\}+\mu_{2} \operatorname{Im}\left\{\lambda^{\prime}\left(\tau_{0}\right)\right\}}{\omega \tau_{0}},
\end{aligned}
$$

which determine the qualities of bifurcating periodic solution in the center manifold at critical value $\tau_{0}$; that is, $\mu_{2}$ determines the direction of the Hopf bifurcation: if $\mu_{2}>0\left(\mu_{2}<\right.$ 0 ), then the Hopf bifurcation is supercritical (subcritical) and the bifurcating periodic solution exists for $\tau>\tau_{0}\left(\tau<\tau_{0}\right) ; \beta_{2}$ determines the stability of the bifurcating periodic solution: the bifurcating periodic solution is stable (unstable) if $\beta_{2}<$ $0\left(\beta_{2}>0\right)$; and $T_{2}$ determines period of the bifurcating periodic solution: the period increases (decreases) if $T_{2}>$ $0(<0)$.

\section{Discussion}

In this paper, we propose a three-dimensional stagestructured predatory-prey model with discrete and distributed delay. Then, by introducing a new variable, the original system is transformed into an equivalent fourdimensional system (4). In Section 2, we analyze the existence and local stability of the positive equilibrium of the system (4). In fact, by use of the Routh-Hurwitz criterion, we know that the positive equilibrium $E^{*}$ is locally asymptotically stable for $\tau=0$. Then, by some computation, we get a threshold $\tau=\tau_{0}$ in Theorem 3. The results indicate that if $\tau<\tau_{0}$, the positive equilibrium $E^{*}$ of system (4) is asymptotically stable; if $\tau=\tau_{0}$, the positive equilibrium $E^{*}$ of system (4) will undergo a Hopf bifurcation. In Section 3, by use of normal form theory and central manifold argument, we establish the formulae for the direction and the stability of the Hopf bifurcation. Our theoretical results show that stage structure and delay play an important role in the dynamics of the system, and delay may lead to complicated dynamic behaviors, such as Hopf bifurcation.

\section{Acknowledgments}

The first author is supported by Postdoctoral Science Foundation of China (no. 2011M501428) and Young Science Funds of Shanxi (no. 2013021002-2). The third author is supported by National Natural Science Foundation of China (no. 11171199). The authors would like to thank the referees of this paper for their helpful comments.

\section{References}

[1] A. J. Lotka, Elements of Physical Biology, Williams \& Wilkins, Baltimore, Md, USA, 1925.

[2] V. Volterra, "Variazioni e fluttuazioni del numero d'individui in specie animali conviventi," Memorie dell'accademia dei Lincei, vol. 2, pp. 31-113, 1926.

[3] B. Liu, Z. Teng, and L. Chen, "Analysis of a predator-prey model with Holling II functional response concerning impulsive control strategy," Journal of Computational and Applied Mathematics, vol. 193, no. 1, pp. 347-362, 2006.

[4] G. Jiang and Q. Lu, "Impulsive state feedback control of a predator-prey model," Journal of Computational and Applied Mathematics, vol. 200, no. 1, pp. 193-207, 2007.

[5] S. Zhang, D. Tan, and L. Chen, "Chaotic behavior of a chemostat model with Beddington-DeAngelis functional response and periodically impulsive invasion," Chaos, Solitons and Fractals, vol. 29, no. 2, pp. 474-482, 2006.

[6] A. Leung, "Periodic solutions for a prey-predator differential delay equation," Journal of Differential Equations, vol. 26, no. 3, pp. 391-403, 1977.

[7] K. Gopalsamy, "Time lags and global stability in two-species competition," Bulletin of Mathematical Biology, vol. 42, no. 5, pp. 729-737, 1980.

[8] X. Wen and Z. Wang, "The existence of periodic solutions for some models with delay," Nonlinear Analysis: Real World Applications, vol. 3, no. 4, pp. 567-581, 2002.

[9] X. Chen, "Periodicity in a nonlinear discrete predator-prey system with state dependent delays," Nonlinear Analysis: Real World Applications, vol. 8, no. 2, pp. 435-446, 2007.

[10] X.-P. Yan and C.-H. Zhang, "Hopf bifurcation in a delayed Lokta-Volterra predator-prey system," Nonlinear Analysis: Real World Applications, vol. 9, no. 1, pp. 114-127, 2008.

[11] S. Ruan, "Absolute stability, conditional stability and bifurcation in Kolmogorov-type predator-prey systems with discrete delays," Quarterly of Applied Mathematics, vol. 59, no. 1, pp. 159$173,2001$.

[12] L.-L. Wang and W.-T. Li, "Existence and global stability of positive periodic solutions of a predator-prey system with delays," Applied Mathematics and Computation, vol. 146, no. 1, pp. 167-185, 2003.

[13] X.-P. Yan and Y.-D. Chu, "Stability and bifurcation analysis for a delayed Lotka-Volterra predator-prey system," Journal of Computational and Applied Mathematics, vol. 196, no. 1, pp. 198210, 2006.

[14] L. Zhou, Y. Tang, and S. Hussein, "Stability and Hopf bifurcation for a delay competition diffusion system," Chaos, Solitons \& Fractals, vol. 14, no. 8, pp. 1201-1225, 2002.

[15] Z. Liu and R. Yuan, "Stability and bifurcation in a harvested one-predator-two-prey model with delays," Chaos, Solitons and Fractals, vol. 27, no. 5, pp. 1395-1407, 2006.

[16] X. Liu and D. Xiao, "Complex dynamic behaviors of a discretetime predator-prey system," Chaos, Solitons \& Fractals, vol. 32, no. 1, pp. 80-94, 2007.

[17] F. Wang and G. Zeng, "Chaos in a Lotka-Volterra predator-prey system with periodically impulsive ratio-harvesting the prey and time delays," Chaos, Solitons and Fractals, vol. 32, no. 4, pp. 1499-1512, 2007.

[18] C. Sun, M. Han, Y. Lin, and Y. Chen, "Global qualitative analysis for a predator-prey system with delay," Chaos, Solitons \& Fractals, vol. 32, no. 4, pp. 1582-1596, 2007. 
[19] C. Çelik, "The stability and Hopf bifurcation for a predator-prey system with time delay," Chaos, Solitons and Fractals, vol. 37, no. 1, pp. 87-99, 2008.

[20] C. Çelik, "Hopf bifurcation of a ratio-dependent predator-prey system with time delay," Chaos, Solitons and Fractals, vol. 42, no. 3, pp. 1474-1484, 2009.

[21] W. Ma and Y. Takeuchi, "Stability analysis on a predator-prey system with distributed delays," Journal of Computational and Applied Mathematics, vol. 88, no. 1, pp. 79-94, 1998.

[22] Z. Teng and M. Rehim, "Persistence in nonautonomous predator-prey systems with infinite delays," Journal of Computational and Applied Mathematics, vol. 197, no. 2, pp. 302-321, 2006.

[23] X. Liao and G. Chen, "Hopf bifurcation and chaos analysis of Chen's system with distributed delays," Chaos, Solitons \& Fractals, vol. 25, no. 1, pp. 197-220, 2005.

[24] C. Çelik, "Dynamical behavior of a ratio dependent predatorprey system with distributed delay," Discrete and Continuous Dynamical Systems B, vol. 16, no. 3, pp. 719-738, 2011.

[25] Y. Song and Y. Peng, "Stability and bifurcation analysis on a logistic model with discrete and distributed delays," Applied Mathematics and Computation, vol. 181, no. 2, pp. 1745-1757, 2006.

[26] Y. Kuang, Delay Differential Equations with Applications in Population Dynamics, Academic Press, Boston, Mass, USA, 1993.

[27] W. G. Aiello and H. I. Freedman, "A time-delay model of singlespecies growth with stage structure," Mathematical Biosciences, vol. 101, no. 2, pp. 139-153, 1990.

[28] R. Xu and Z. Wang, "Periodic solutions of a nonautonomous predator-prey system with stage structure and time delays," Journal of Computational and Applied Mathematics, vol. 196, no. 1, pp. 70-86, 2006.

[29] J. Jiao, L. Chen, and S. Cai, "A delayed stage-structured Holling II predator-prey model with mutual interference and impulsive perturbations on predator," Chaos, Solitons and Fractals, vol. 40, no. 4, pp. 1946-1955, 2009.

[30] C. Wei and L. Chen, "Eco-epidemiology model with age structure and prey-dependent consumption for pest management," Applied Mathematical Modelling, vol. 33, no. 12, pp. 4354-4363, 2009.

[31] X. Meng, J. Jiao, and L. Chen, "The dynamics of an age structured predator-prey model with disturbing pulse and time delays," Nonlinear Analysis: Real World Applications, vol. 9, no. 2, pp. 547-561, 2008.

[32] H. Zhang, L. Chen, and J. J. Nieto, "A delayed epidemic model with stage-structure and pulses for pest management strategy," Nonlinear Analysis: Real World Applications, vol. 9, no. 4, pp. 1714-1726, 2008.

[33] R. Shi and L. Chen, "Staged-structured Lotka-Volterra predatorprey models for pest management," Applied Mathematics and Computation, vol. 203, no. 1, pp. 258-265, 2008.

[34] J. M. Cushing, Integro-Differential Equations and Delay Models in Population Dynamics, Springer, Berlin, Germany, 1977.

[35] B. D. Hassard, N. D. Kazarinoff, and Y. H. Wan, Theory and Applications of Hopf Bifurcation, Cambridge University Press, Cambridge, UK, 1981. 


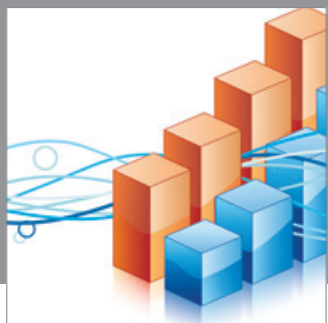

Advances in

Operations Research

mansans

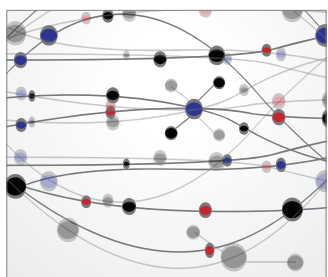

The Scientific World Journal
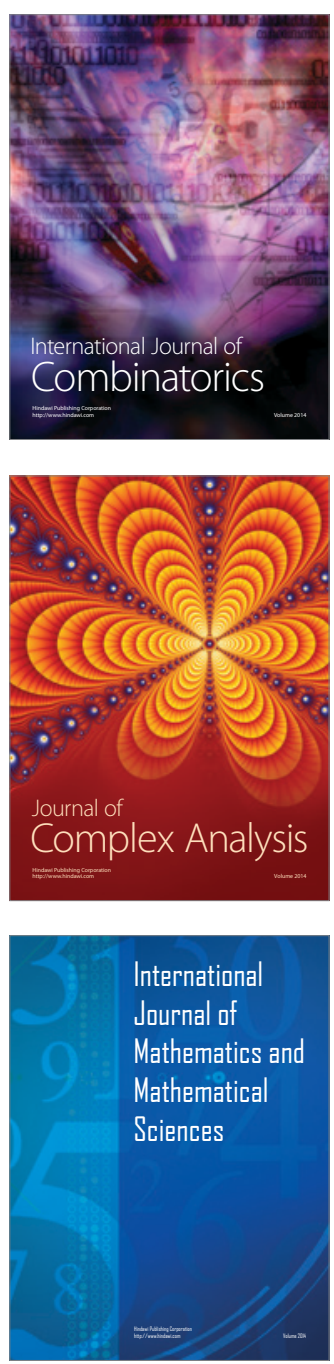
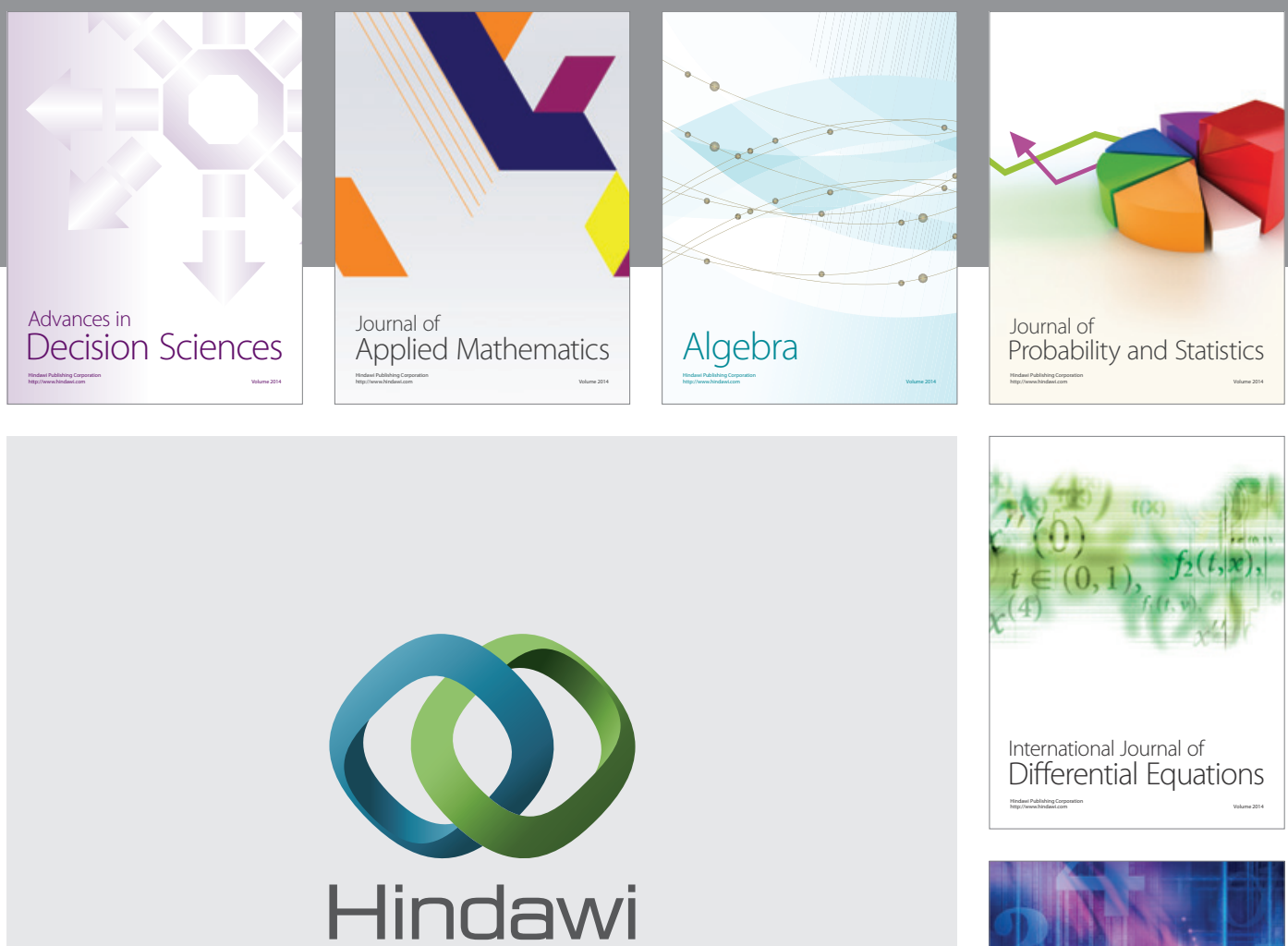

Submit your manuscripts at http://www.hindawi.com
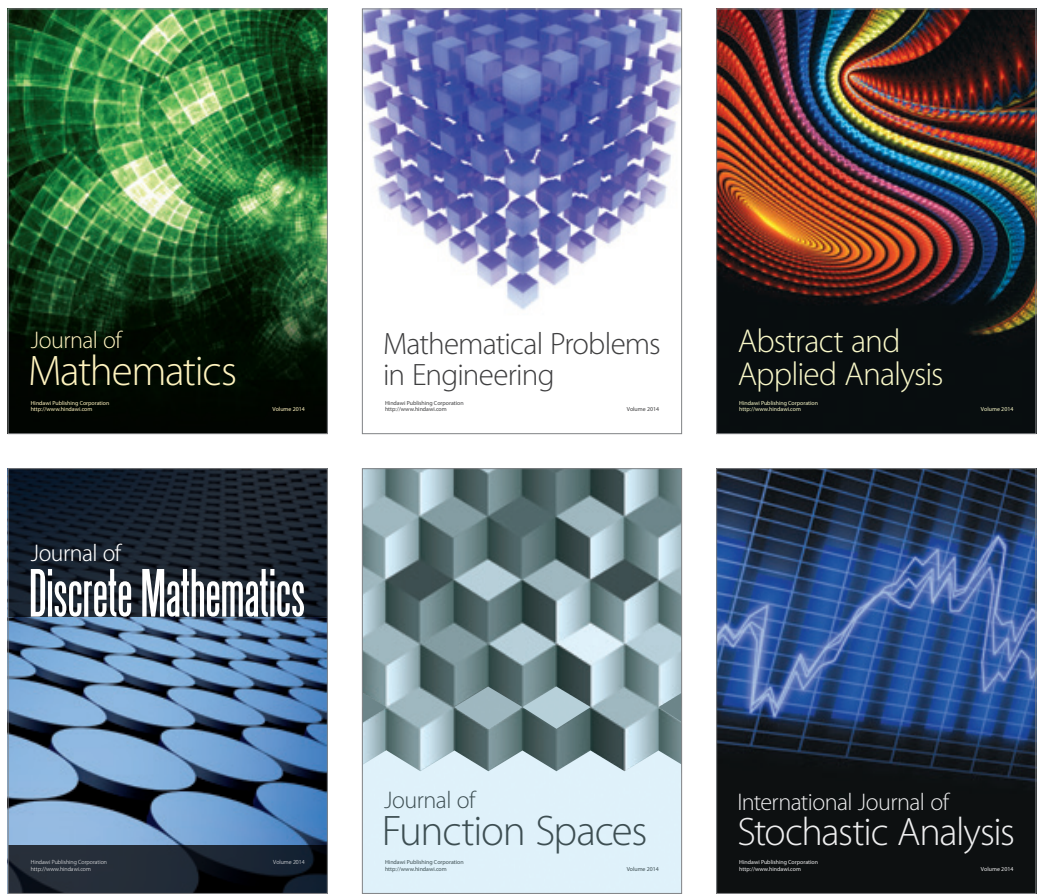

Journal of

Function Spaces

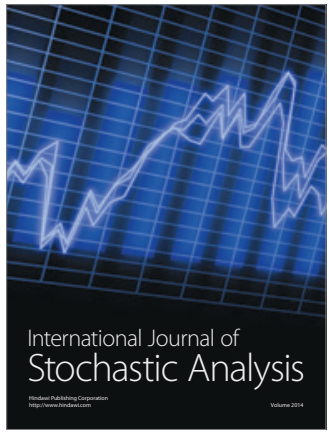

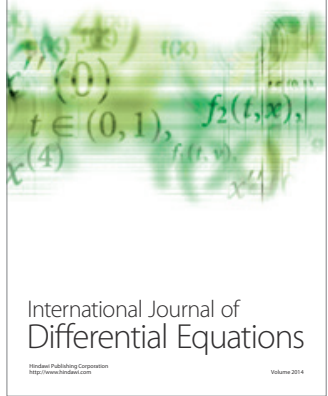
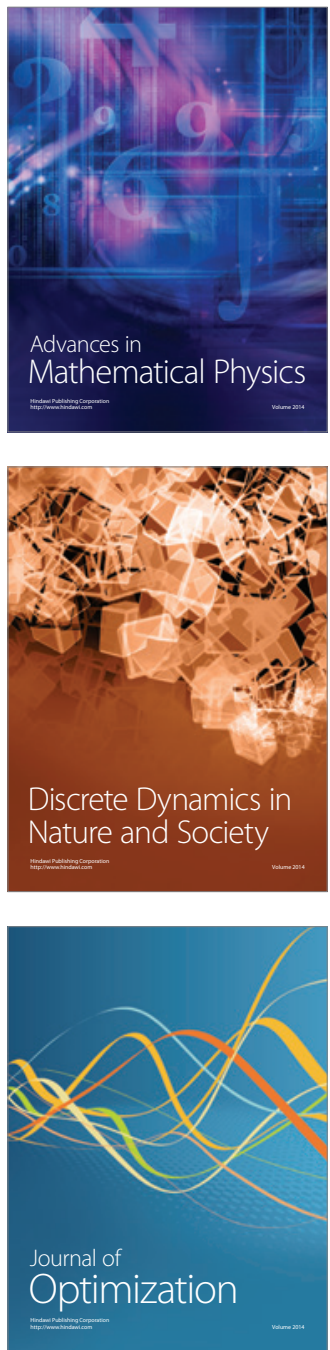